\title{
Oscillatory-shear-induced order in nonaqueous dispersions of charged colloidal spheres
}

\author{
Y.D. Yan, J.K.G. Dhont ${ }^{1}$, C. Smits and H.N.W. Lekkerkerker \\ Van 't Hoff Laboratory for Physical and Colloid Chemistry, University of Utrecht, \\ Padualaan 8, $3584 \mathrm{CH}$ Utrecht, The Netherlands
}

Received 20 July 1993

\begin{abstract}
A light scattering study is presented of the nonequilibrium microstructure of nonaqueous dispersions of repulsive charged colloidal silica spheres subjected to an oscillatory shear flow. Two ordered structures are induced upon increasing the oscillation amplitude: alternating face-centred-cubic (fcc) twins and registered randomly stacked sliding layers. The transition between these structures shifts to lower amplitudes as particle volume fraction is increased. This is consistent with predictions of a simple strained fcc-lattice packing model previously developed for hard spheres. There are, however, quantitative differences due to the softness of the interparticle interactions of our system. In addition, we find that the fcc twins appear much more slowly than the sliding layers and that the former occurs mainly due to shear orientation of existing crystallites. We also observe that the formation of the sliding layers depends on the amplitude as well as the frequency of oscillation.
\end{abstract}

\section{Introduction}

The Péclet number $\mathrm{Pe}$ is an important measure of the strength of the convective motion relative to diffusive motion in a sheared colloidal dispersion. It is defined as $\mathrm{Pe}=\dot{\gamma} a^{2} / D_{0}=6 \pi \eta_{0} a^{3} \dot{\gamma} / k T$, where $\dot{\gamma}$ is the shear rate, $a$ is the particle radius, $D_{0}$ is the Stokes-Einstein diffusion coefficient at infinite dilution, $\eta_{0}$ is the solvent viscosity, and $k T$ is the thermal energy. For $\mathrm{Pe} \gg 1$, hydrodynamic force dominates thermodynamic forces so the dispersion microstructure is expected to be significantly distorted from its equilibrium state. Two simple flow fields are of particular interest: steady-state and oscillatory shears.

Whereas existing experimental evidence indicates that the nonequilibrium microstructure of a dispersion undergoing a high steady-state shear flow is correlated to the microscopic order at rest $[1,2]$, there are results which show

\footnotetext{
${ }^{1}$ To whom correspondence should be addressed.
} 
that an oscillatory shear flow can induce crystalline-like interparticle ordering even in a dispersion which is a liquid in equilibrium. Work reported on the latter case is, however, rather limited.

Oscillatory-shear-induced structural ordering was first reported by Scott et al. [3]. They found that after about 100 cycles of repeated shearings at certain oscillation amplitudes, the mean packing density of small steel balls initially in random loose packing levelled off above the random close packing limit, indicating the presence of regions of shear-induced crystalline order. Recently, a more detailed study has been made by Ackerson and Pusey [4,5] for sterically stabilized hard-sphere-like polymethylmethacrylate (PMMA) latex sphere dispersions. It was found that over a certain range of the oscillation amplitude, alternating twin face-centred-cubic ( $\mathrm{fcc}$ ) structures and registered randomly stacked sliding layers could be induced. The transition between these structures depends on both the oscillation amplitude and the particle volume fraction. A simple geometric packing model based on a strained fcc lattice has been used to elucidate this transition.

In this study, we extend these oscillatory shear investigations to a different model colloidal system in an attempt to gain more insight into the microstructural response to such a flow field. The dispersions we used are comprised of coated monodisperse silica spheres having medium-range repulsive potential (due to surface charges) and dispersed in a close optical matching mixture of ethanol and toluene. Apart from those orderings found also for hard-sphere dispersions, we present some additional features. Our results will be compared with those from hard-sphere systems and discussed in the light of the abovementioned geometric packing model.

\section{Experimental}

\subsection{Particle synthesis and characterization}

The model particles used here are silica spheres sterically stabilized with $\gamma$-methacryloxypropyltrimethoxysilane (TPM). The silica cores were prepared in a condensation polymerization reaction of tetraethoxysilane in ethanol and ammonia according to the method described by Stöber et al. [6]. The surface coating was achieved through the reactions of the surface silanol groups of the silica cores with the silane coupling agent TPM. The coated layer has a thickness of about 2 to $4 \mathrm{~nm}$. A detailed description of the coating procedure can be found elsewhere [7]. The resulting particles are referred to here as TPM-silica.

These particles are stable in weakly polar organic solvents such as ethanol 
Table I

Characteristics of the TPM-silica dispersions.

\begin{tabular}{lll}
\hline Solvent & composition & $79 / 21(\mathrm{v} / \mathrm{v})$ toluene/ethanol \\
& refractive index $\left(632.8 \mathrm{~nm}, 20^{\circ} \mathrm{C}\right)$ & $n_{0}=1.4664$ \\
& density $\left(20^{\circ} \mathrm{C}\right)$ & $\rho_{0}=0.8481 \mathrm{~g} \mathrm{~cm}^{-3}$ \\
& viscosity $\left(20^{\circ} \mathrm{C}\right)^{\mathrm{a}}$ & $\eta_{0} \approx 0.679 \mathrm{cP}$ \\
& dielectric constant & $\varepsilon \approx 10$ \\
TPM-silica & optical radius & $a=470.4 \mathrm{~nm}$ \\
& relative standard deviation in radius from TEM & $\sigma=3 \%$ \\
& mass density & $\rho=1.91 \mathrm{~g} \mathrm{~cm}^{-3}$ \\
& elementary charges per particle & $Q \approx 1300$ \\
& Debye screening length $(\mathrm{at} \phi=0)^{\mathrm{c}}$ & $\kappa_{0}^{-1} \approx 60 \mathrm{~nm}$ \\
\hline
\end{tabular}

${ }^{\mathrm{a}}$ From ref. [2].

${ }^{\mathrm{b}}$ Measured by first weighing $25 \mathrm{ml}$ concentrated dispersion and then drying and weighing a known amount of this sample.

${ }^{\mathrm{c}}$ For low electrolyte concentrations $[8], \kappa^{2}=\kappa_{0}^{2}+e^{2} C_{p} / \varepsilon \varepsilon_{0} k T$ with $C_{p}\left[\right.$ ions $\left./ \mathrm{m}^{3}\right]=[1 /(1-\phi)]$ $3 \phi Q / 4 \pi a^{3}$.

and toluene. In this work we dispersed them in a mixture of these two. The solvent composition (see table I) was so chosen that its refractive index matches closely that of the particles so that multiple light scattering effects were weak even in concentrated dispersions. In this solvent the particles are negatively charged due to the dissociation of the acid silanol groups on the silica core surface and those of the TPM-oligomers attached to the surface [7]. The surface charge of the particles and the Debye screening length of the dispersion solvent were estimated from results on similar TPM-silica systems of earlier studies $[7,8]$ and are listed in table $I$.

Particle size was determined using both light scattering and transmission electron microscopy (TEM) on a very dilute dispersion in ethanol. The optical radius was measured by static light scattering at $25^{\circ} \mathrm{C}$, using a Fica-50 photometer operating at $546 \mathrm{~nm}$. The results are summarized in table I along with the measured mass density of the particles.

The weight fraction of a concentrated stock sample was determined by drying and weighing. The corresponding particle volume fraction $(\phi)$ was calculated using the densities of the dispersion solvent and the TPM-silica.

\subsection{Parallel-plate shear set-up and light scattering geometry}

The same light scattering shear apparatus as used in our previous study [2] was employed in this experiment, except that some changes were made to accommodate oscillatory shear. Fig. 1a gives a schematic illustration of this set-up. It is composed of two main parts: a parallel-plate shear cell and a two-dimensional CCD camera. In operation an unpolarized $\mathrm{He}-\mathrm{Ne}$ laser beam $(632.8 \mathrm{~nm}, 5 \mathrm{~mW})$ is incident, in a direction normal to the parallel plates, onto 
(a)

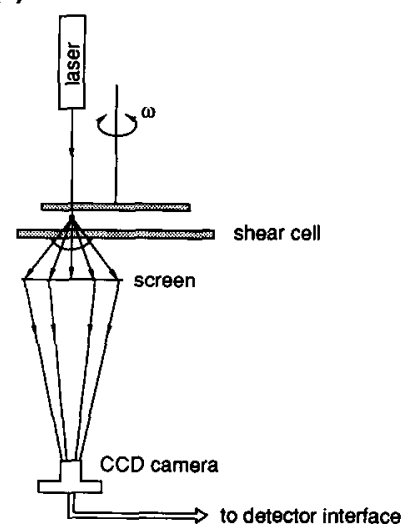

(b)

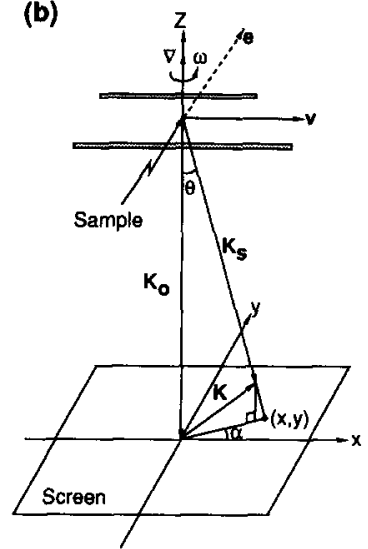

Fig. 1. (a) Overview of the parallel-plate light scattering shear set-up used in this work. (b) Scattering geometry corresponding to one of the two oscillating directions. $V$ is the shear velocity along the $x$-axis. The velocity gradient $\nabla$ is along $z$, and the vorticity $e$ is defined as $\hat{e}=\hat{\nabla} \times \hat{V}$ and is thus along $y$. The velocity-vorticity plane ( $x y$-plane) is horizontal but normal to the paper. $k$ $\left(\boldsymbol{k}=\boldsymbol{k}_{s}-\boldsymbol{k}_{0}\right)$ is the scattering wavevector at a point $(x, y)$ on the screen. $\theta$ is the scattering angle and $\alpha$ is the polar angle of the data point $(x, y)$.

the sample, which is kept in between the upper oscillating and bottom stationary plates. The scattered light from the sample, being the product of the particle form factor and static structure factor, is projected on a flat screen of translucent paper normal to the incident beam, and the image is then taken using the CCD camera placed beneath the screen.

Full details about the CCD camera and the shear cell have been described previously [2]. Here we give a brief description of the shear cell. The two round plates holding the sample are made of fused silica. The upper plate has a smaller radius of $50 \mathrm{~mm}$ and can exhibit sinusoidally oscillating displacement. The laser beam passes through the sample at a point $32.4 \mathrm{~mm}$ from the centre of the oscillating plate. The sample gap spacing is fixed at $0.5 \mathrm{~mm}$. In order to avoid refraction of the scattered light at the plate-air interface, we glued a partial hemispherical plano-convex lens to the bottom plate. The lens is designed such that the centre of the radius of curvature of the lens coincides with the centre of the scattering volume. The sample-to-screen and screen-tocamera distances (all adjustable) are about $4.8 \mathrm{~cm}$ and $68 \mathrm{~cm}$, respectively. So in the present case the maximum scattering angle detectable by the camera is about $45^{\circ}$, which corresponds to $(\mathrm{ka})_{\max } \approx 5.2$ in this work.

Since the incident laser beam is parallel to the shear-gradient direction, for small angles the scattering wavevectors probe the flow-vorticity plane. The flow geometry and the directions of the scattering wavevectors of this experiment are illustrated in fig. 1b. At a point $(x, y)$ on the screen (or 
equivalently, a pixel on the camera), corresponding to a scattering wavevector $\boldsymbol{k}$, the three respective $\boldsymbol{k}$-components along the velocity, shear, and vorticity directions can be calculated following ref. [2], except that here the scattering angle $\theta$ is given by $\theta=\tan ^{-1}\left[0.012\left(x^{2}+y^{2}\right)^{1 / 2}\right]$.

All scattering measurements reported in the next section were performed at a temperature of $20 \pm 1^{\circ} \mathrm{C}$.

\section{Results and discussion}

\subsection{Structures in equilibrium}

The equilibrium phase behaviour of our TPM-silica dispersions was examined. This was done by concentrating or diluting in steps of $\phi \approx 0.7 \%$, in a $1 \mathrm{~cm}$ diameter cuvette, a concentrated dispersion (taken from a previously prepared stock sample) of known particle volume fraction. After each step, the sample was thoroughly homogenized by using a Vortex mixer. Visual inspection of the crystallization process was made by illuminating the sample obliquely from behind by a broad beam of white light.

We examined $\phi$ up to $49.4 \%$, the freezing concentration expected for hard spheres in computer simulations [9]. Because of the large size of our particles and also the big difference in density between the particles and the dispersion solvent, significant particle sedimentation upon sample standing was evident $(\sim 0.6 \mathrm{~mm} / \mathrm{hr}$ at $\phi \approx 8 \%)$. No crystallization was observed in the bulk of our samples. Instead, a thin layer of small crystallites was visible only in the interface between the supernatant and the sedimenting dispersion phase. Since the range of concentration over which relatively fast homogeneous nucleation can take place is very narrow $[8,10,11]$ and crystal nucleation rates may also be reduced by particle sedimentation effects, bulk crystallization was probably missed in our experiments. The optimum concentration profile for fast nucleation can, however, develop in the interface during or after particle sedimentation [12]. Nevertheless, from the phase behaviour of similar but smaller TPM-silica dispersions $[2,8,12]$, we expect our system to freeze around $\phi=20 \%$.

\subsection{Structures under oscillatory shear}

After injection into the shear cell, samples were randomized by vigorously rotating the upper plate. A diffuse featureless first-order Debye-Scherrer (DS) ring was visible upon a short period of sample standing, characteristic of the amorphous nature of these samples.

Like in previous studies on hard-sphere dispersions [4,5], we first examine 
the effects of the strain amplitude (i.e., the amplitude of oscillation at the scattering point divided by the sample gap spacing) on the dispersion microstructure at various particle volume fractions. A strain amplitude of unity implies relative mechanically driven displacement of two particles in the flow direction equal to their separation in the shear direction. For these experiments the oscillation frequency was fixed at $5.0 \mathrm{~Hz}$ and the accessible strain amplitude ranged from 0 to 5.8 , which results in the Péclet numbers during an oscillation cycle between 0 and 60 . Samples were randomized before each run.

For particle concentrations $\phi<\sim 8 \%$, liquid-like scattering behaviour (cf. fig. 2a) was observed over the full range of the accessible strain amplitudes. At higher concentrations, a featureless DS ring was visible at very small strain amplitudes and it oscillated with the same period as the applied stress. However, a six-fold streak pattern was induced on further increasing the strain amplitude as shown in fig. $2 \mathrm{~b}$. The streaks were oscillating during a cycle. Moreover, we found that the streaks were made up of two three-fold mirrorimage twins. Figs. $2 \mathrm{c}$ and $2 \mathrm{~d}$ illustrate these three-folds obtained at the two extremes of a cycle. As the strain amplitude was raised to larger values, a hexagonally spaced six-spot pattern was formed (fig. 2e). These spots were quite stationary and are about 30 degrees rotated with respect to the above six-fold streak pattern. The transition amplitude between the six-fold streaks and the six-fold spots decreased with increasing particle concentration. Fig. 3 summarizes our findings. These phenomena seem to be in essential agreement with those found for hard-sphere dispersions by Ackerson and Pusey [4,5] and in the latter case have been explained by Ackerson [5] in terms of a geometric packing model for homogeneous oscillatory strain of an fcc crystal, similar in spirit to the steady-state shear flow model proposed by Hoffman [13].

According to this geometric packing model, in hard-sphere dispersions the two three-fold twin scattering patterns were the result of particle ordering under shear into two oscillating fcc twins. Figs. $4 \mathrm{a}$ and $4 \mathrm{~b}$ depict the respective real space lattice structures corresponding to the scattering patterns of figs. $2 \mathrm{c}$ and $2 \mathrm{~d}$. One set of (111) lattice planes lies in planes of constant velocity parallel to the surfaces of the cell plates and is most densely packed along the vorticity axis. Relatively easy transition between the two twin fcc structures can occur at small strain amplitudes $(\sim 1)$; at larger amplitudes structural reorganization has to take place in order to allow more extensive motion of a particle in the flow direction. The fact that the Bragg peaks are streaks rather than spots indicates that the close packed axes of the aligned (111) planes are distributed about the vorticity axis. This would allow a greater strain amplitude than that for a crystal with perfect alignment with the vorticity direction.

As is further known from this model, the six-fold spot scattering pattern in hard-sphere systems was due to registered randomly stacked layers of particles 

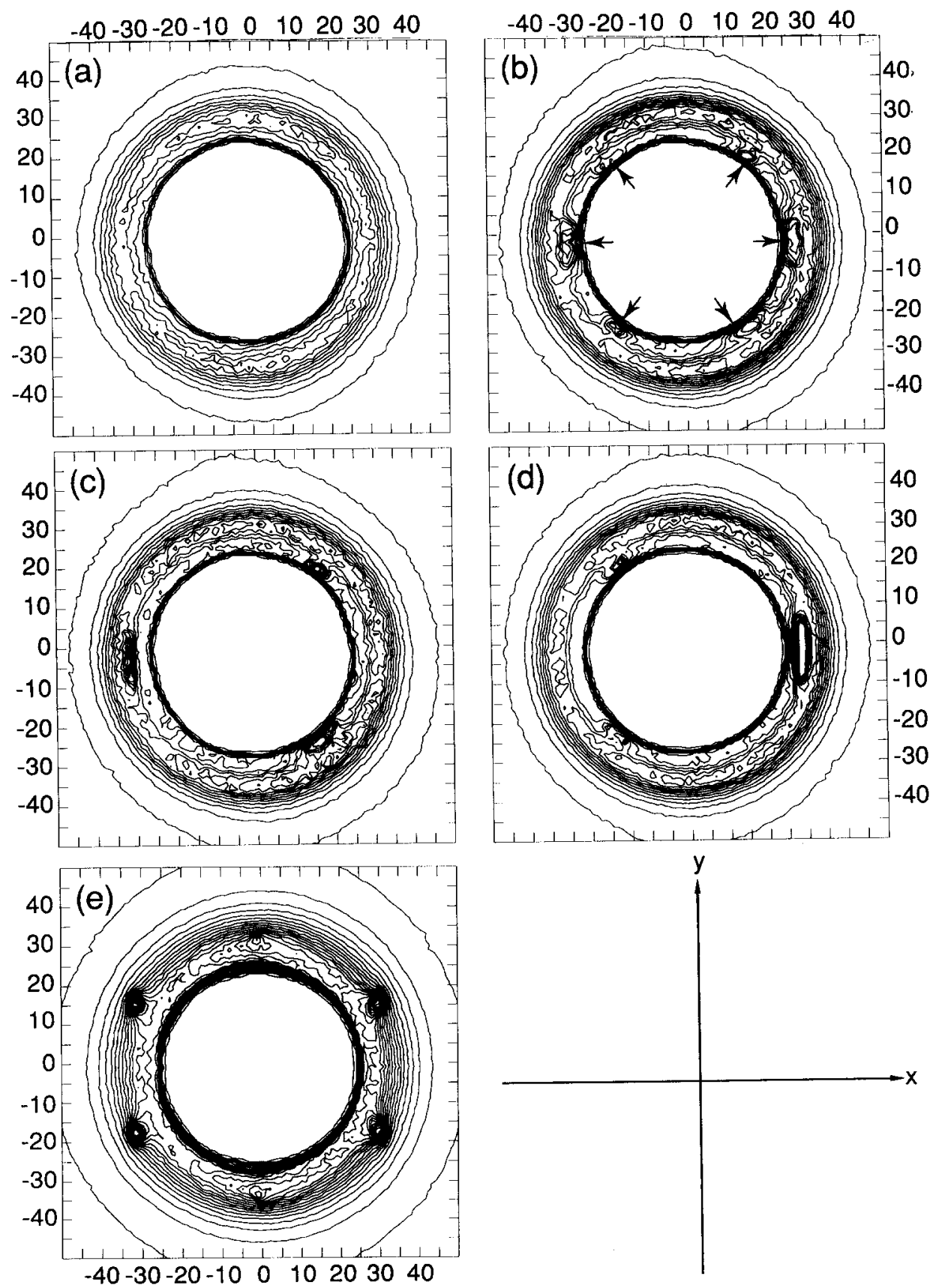

Fig. 2. Light scattering intensity contour patterns in the velocity-vorticity or $x y$-plane (see the indication at the bottom-right corner) with increasing strain amplitude, $A$, for a dispersion of $\phi=8.07 \%$ sheared for about $30 \mathrm{~min}$ at a fixed oscillation frequency of $5.0 \mathrm{~Hz}$. (a) At rest. (b) Six-fold streaks at $A=0.5$. Arrows are drawn to guide the eye. (c),(d) The corresponding three-fold twin streak patterns of (b) at the two extremes of an oscillation cycle. (e) Six-fold spots at $A=5.0$. Note that the velocity and vorticity axes are in units of data points from which the corresponding scattering wavevectors can be calculated. The central areas in these plots are blocked out by the beamstop. 


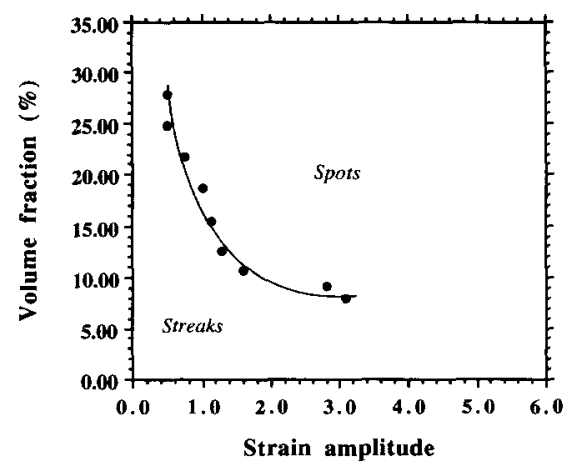

Fig. 3. Transition between the six-fold streak pattern of scattering and the six-fold spot pattern with increasing strain amplitude as a function of particle volume fraction at an oscillation frequency of $5.0 \mathrm{~Hz}$. The data points indicate the first time the spot patterns were observed. The best visual fit is drawn to guide the eye.

(a)

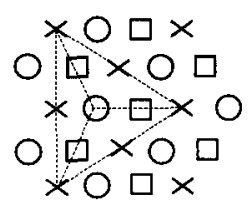

(b)

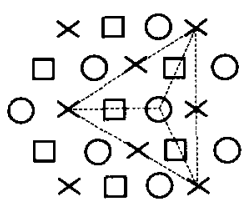

(c)

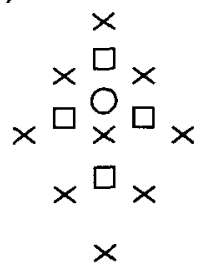

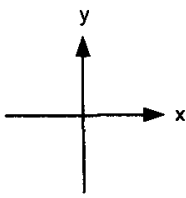

Fig. 4. Geometric packing of particles at small and larger strain amplitudes. (a), (b), and (c) lead to the scattering patterns (c), (d), and (e) in fig. 2, respectively. Here (O) represents a particle in the top layer, $(\square)$ in the middle, and $(x)$ in the bottom. Some particles are connected by dashed lines to show the geometry of the fcc twins.

under shear. In contrast to the twin fcc packing, the close packed direction within a layer in the randomly stacked structures is oriented along the flow axis as plotted in fig. 4c. Distribution of this orientation about its average would only lower the strain amplitudes allowed for the existence of randomly stacked layers. As a result, one observes the spot pattern in scattering.

On the basis of this model, Ackerson has argued that layers of particles in the fcc structures can slip freely one over another at particle volume fractions below $40 \%$. Increasing the volume fraction above this value, only transition between the two fcc twins is still possible in a limited region, but this transition becomes more and more hindered, resulting in a decrease of the allowed strain amplitude. At $\phi=64 \%$, particles are simply caged in their nearest neighbours and no transition is possible without introducing particle displacements other than those caused by the shear flow. If particles are on the other hand aligned with close packed rows along the flow direction, stacked layers may then slip freely over each other up to $\phi=58 \%$. Above this value, the layers may still 
slip over each other performing a zig-zag motion up to $\phi=64 \%$, where particles are again caged. The flow induced centring between different particle layers makes the layer movement possible at large strain amplitudes up to $\phi=64 \%$. Particle motion finally ceases at the closest packing fraction of $74 \%$.

Therefore, it becomes obvious that transition from the fcc twins to sliding layers may occur on increasing the strain amplitude and that the transition shifts to lower amplitudes at higher particle volume fractions. Although our dispersions are quite different from those of hard spheres and generally have much lower packing fractions than required in the above model, these ideas appear to be, at least qualitatively, applicable. This can perhaps be understood by taking into account the excluded volume effect of the electric double layers of our particles; on scaling to effective hard spheres, the soft repulsive potential of our particles leads to an extension of their hard-core radius. Quantitatively, deviations from the hard-sphere model are expected for charged systems due to the softness of the interparticle interactions. One such example is that the "caging" effect may not be as strong as that seen in the hard-sphere packing, so in soft interacting systems the shear-induced transition between the fcc twin structures and the sliding layers may proceed to smaller strain amplitudes than the estimated lower limit of 0.9 at $\phi=64 \%$ in hard spheres [5]. This can be clearly seen in fig. 3 .

It is interesting to speculate that the aforementioned shear-induced structures may form as well in very dilute but strongly interacting charge-stabilized dispersions. The basic ideas on which the geometric packing model relies are, however, questionable for these dilute systems. In a recent Brownian dynamics simulation of charged polystyrene latex spheres subjected to a similar oscillatory shear flow to what we used here, Xue and Grest [14] found strong shear-induced string ordering of particles along the flow direction at system temperatures very close to the melting point. However, no ordering effects like those seen in this paper were observed even when starting the simulation with particles on an fcc lattice. This is perhaps not surprising as their accessed Péclet numbers were rather small.

We noticed that the six-fold streak pattern appeared to be more fragile than the six-fold spot pattern. A six-fold streak pattern formed at smaller amplitudes could be gradually transformed into a six-fold spot pattern if the strain amplitude was raised to a sufficiently high value, but not vice versa. Here the amplitude was changed in succession without rerandomizing the samples. This is in accordance with observations from hard-sphere dispersions [5] and shows the shear history dependence of the nonequilibrium microstructure.

Having discussed the basic ordering phenomena of our system, we now turn to some additional features. First of all, we noticed that occurrence of the six-fold streak pattern (fig. 2b) was much slower than that of the six-fold spot 
pattern (fig. 2e). A test experiment was carried out with a dispersion of $\phi \approx 8 \%$. Without resting the sample after its randomization, the streak pattern developed in about $17 \mathrm{~min}$ of oscillation, whereas the spot pattern appeared within only 1 or 2 min after shearing the sample. More interestingly, we also found that the shearing time required for the formation of the streak pattern was initially dependent on the time of sample resting after randomization, with the sum of these two times being however almost constant. After a sufficient period of sample equilibration before subject to oscillation, the shearing time needed decreased to a nearly constant, lowest value. These results are presented in table II.

We also noticed that the streaks formed at scattering angles smaller than that of the coexisting DS ring (cf. fig. 2b) but coincided with the speckles which appeared in about $45 \mathrm{~min}$ of sample resting. This coexisting ring obviously resulted from a denser particle sediment which formed during the long experimental duration. The speckles must be an indication of crystallite formation when the sample was left to equilibrate undisturbed (cf. section 3.1).

Therefore, we deduce with the help of table II that in the above test dispersion, equilibrium crystal nucleation must have started (in the interface) ca $14 \mathrm{~min}$ after sample randomization. Once equilibrium crystallites were formed, only about $3 \mathrm{~min}$ (or equivalently, 900 cycles) of oscillation were needed to orient them. The scattering intensity of these initially randomly oriented crystallites was thus focused onto six streaks and became more readily distinguished. We believe that the fcc-twin formation in this study is mainly the consequence of the orienting effect of the shear flow on existing crystallites. However, one cannot rule out the possibility that oscillatory-shear-induced nuclei were also present but perhaps they had not grown to have a sizeable effect on scattering within the first $17 \mathrm{~min}$ of shearing. It is not clear as to whether this long nucleation time of shear-induced fcc crystallites was simply

Table II

Shearing time, $t_{\text {shear }}$, required for the formation of the six-fold streak scattering pattern in a dispersion of $\phi=8.07 \%$, showing its dependence on sample resting time after randomization, $t_{\text {rest }}$. The strain amplitude and oscillation frequency were fixed at 0.5 and $5.0 \mathrm{~Hz}$, respectively. The last column gives $t_{\text {total }}=t_{\text {rest }}+t_{\text {shear }}$.

\begin{tabular}{ccc}
\hline$t_{\text {rest }} / \min$ & $t_{\text {shear }} / \min$ & $t_{\text {total }} / \min$ \\
\hline 0 & 17 & 17 \\
5 & 11.5 & 16.5 \\
10 & 7.9 & 17.9 \\
17 & 3 & $(20)$ \\
30 & 2.5 & $(32.5)$ \\
60 & 3.5 & $(63.5)$ \\
\hline
\end{tabular}


due to particle sedimentation under gravity, although it is intuitively expected that three-dimensional ordering is more susceptible to particle settling than two-dimensional layering. It should be mentioned that since the sliding layers in this study were formed within a very short period of oscillation after sample randomization, they were generally free from particle settling effects. Noticeably, in the very slowly sedimenting hard-sphere-like PMMA dispersions [5], both the fcc and layer scattering patterns were observed within 5 min of oscillation, well before the formation of equilibrium nuclei which was estimated to be ca $30 \mathrm{~min}$ at $\phi=55.3 \%$. Use of a Couette shear cell may alleviate particle sedimentation effects since particles will then have to sediment over a long distance to affect the concentration in the scattering volume. It may thus enable one to discern purely shear-induced fcc crystals.

Our second new observation is the dependence of the sliding layer formation on oscillation frequency. We found that upon increasing the frequency, the transition between the alternating fcc twins and the registered randomly stacked layers shifted to lower strain amplitudes (fig. 5). The lower amplitude limit was, however, still roughly governed by geometric packing requirements. As can also be seen from fig. 5, there appears to be a lower limit in the oscillation frequency below which no sliding layer structures can be induced. This is perhaps not difficult to understand since if the frequency is lower to, say, $1 \mathrm{~Hz}$, the maximum Péclet number for a strain amplitude of 5.8 is reduced from 60 at $5.0 \mathrm{~Hz}$ to 12 . Therefore, the hydrodynamic force is significantly reduced. Furthermore, for very slow oscillating motions the maximum Péclet number during a cycle can no longer be used to indicate the relative strength of the hydrodynamic force exerted on the particles, so the natural Brownian relaxation $(\sim 0.3 \mathrm{~s}$ for our samples) and direct interparticle interactions may well restore the quiescent-state microstructure.

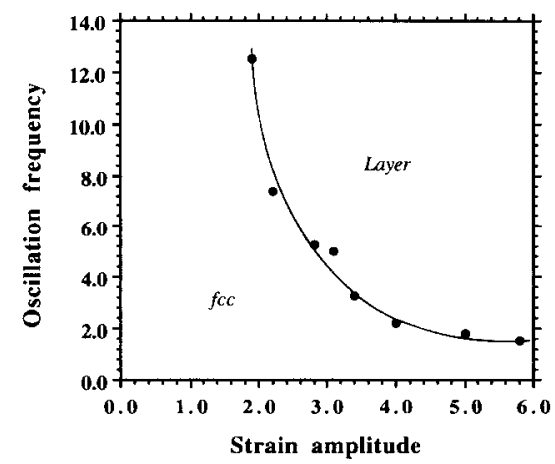

Fig. 5. Transition between the alternating fcc twins and the sliding layers as a function of both the strain amplitude and oscillation frequency in a dispersion of $\phi=8.07 \%$. The best visual fit is drawn to guide the eye. 
Finally, higher-order shear-induced Bragg peaks were sometimes visualized on the detection screen, but they were very weak due to the deep first minimum in the particle form factor beyond the first-order DS ring. Moreover, the scattering intensity of the first-order structures became immediately less intense when the shear flow was stopped, and weak peaks simply vanished.

\section{Conclusions}

We have extended the study of oscillatory shear effect on dispersion microstructure to a new colloidal system in which particles interact via a screened Columb potential. It is shown that the oscillatory-shear-induced structural orderings in this system bear essential resemblance to those found in very different, dense hard-sphere dispersions. With increasing oscillation amplitude two ordered structures are evident: oscillating fcc twins and registered randomly stacked sliding layers. For both systems, the transition between the fcc twins and the sliding layers shifts to lower amplitudes at higher particle concentrations. This can be explained on the basis of a hard-sphere geometric packing model, although quantitative deviation is noticed in our system due to the softness of the interparticle interactions.

The following additional features have been explored in this study. Firstly, we notice that formation of the twin fcc structures is much slower than that of the sliding layers and the former may be influenced by particle settling under gravity. We believe that in our experiment the fcc twin formation is not merely effected by the application of the oscillatory shear, but oscillation is at least necessary to orient existing crystallites. On the other hand it appears that the existence of the sliding layers is simply due to the effect of the oscillatory shear flow. Secondly, we find that the induction of the sliding layers depends not only on the strain amplitude but on the oscillation frequency as well. No layering can be induced below either certain amplitude or certain frequency of oscillation.

Layer-like orderings can be easily induced in our liquid-like dispersion of $\phi \approx 8 \%$ by an oscillatory flow. By contrast, no orderings are found in this sample when subject to a steady-state flow of accessible Péclet number up to 555. Clearly, repeated interactions of a particle with its nearest neighbours enhance structure formation.

\section{Acknowledgements}

One of us (YDY) wishes to thank Chellappah Pathmamanoharan for his advice in the sample preparation, and Jeroen S. van Duijneveldt for several 
valuable discussions. We are grateful to Ingrid van Rooijen for reproducing some of the figures. This work is part of the research programme of the Foundation for Fundamental Research of Matter (FOM) with financial support from the Netherlands Organization for Pure Research (NWO).

\section{References}

[1] B.J. Ackerson, Physica A 174 (1991) 15.

[2] Y.D. Yan and J.K.G. Dhont, Physica A 198 (1993) 78.

[3] G.D. Scott, A.M. Charlesworth and M.K. Mak, J. Chem. Phys. 40 (1964) 611.

[4] B.J. Ackerson and P.N. Pusey, Phys. Rev. Lett. 61 (1988) 1033.

[5] B.J. Ackerson, J. Rheol. 34 (1990) 553.

[6] W. Stöber, A. Fink and E. Bohn, J. Colloid Interface Sci. 26 (1968) 62.

[7] A.P. Philipse and A. Vrij, J. Colloid Interface Sci. 128 (1989) 121.

[8] J.K.G. Dhont, C. Smits and H.N.W. Lekkerkerker, J. Colloid Interface Sci. 152 (1992) 386.

[9] W.G. Hoover and F.H. Ree, J. Chem. Phys. 49 (1968) 3609.

[10] P.N. Pusey and W. van Megen, in: Physics of Complex and Supramolecular Fluids, S.A. Safran and N.A. Clark, eds. (Wiley, New York, 1987) 673.

[11] W.B. Russel, Phase Transitions 21 (1990) 127.

[12] J.S. van Duijneveldt, J.K.G. Dhont and H.N.W. Lekkerkerker, to appear in J. Chem. Phys.

[13] R.L. Hoffman, Trans. Soc. Rheol. 16 (1972) 155.

[14] W. Xue and G.S. Grest, Phys. Rev. Lett. 64 (1990) 419. 